\title{
Does Calcium Turn a Zwitterionic Lipid Cationic?
}

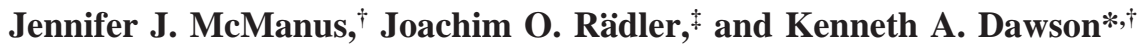 \\ Department of Chemistry, University College Dublin, Belfield, Dublin 4, Ireland, and Ludwig Maximillian \\ University, Department of Experimental Physics, Gesschwister-Scholl-Platz 1, D-80539 Munich, Germany
}

Received: February 24, 2003; In Final Form: June 23, 2003

The structure of a complex formed from DNA, calcium, and DPPC has been studied by small-angle X-ray scattering (SAXS). Previous DSC and temperature scanning ultrasound have shown that in excess lipid two DPPC phases exist, one corresponding to DNA bound lipid and the other uncomplexed lipid, not directly involved in complex formation. ${ }^{1,2}$ We have investigated the effect of calcium on both phases within the complex structure. Contrary to the observations in the ternary DPPC/calcium/water system, the binding of calcium to the DPPC membrane in this quaternary system containing DNA appears to be significantly enhanced. We have estimated the stoichiometry of calcium binding within the complex, and we concluded that the zwitterionic lipid is directly involved in binding to DNA.

\section{Introduction}

Due to their potential for use as gene delivery devices, considerable attention has been paid to complexes composed of DNA and cationic lipids, generally termed lipoplexes in both theoretical and experimental investigations. ${ }^{3}$ This work has been conducted over two decades, prompted by observations of Felgner et al. 4,5 Their main drawback is the associated cytotoxicities with some formulations and immunogenic effects that lead to limitations in dose. Much of the research carried out has been on model DNA complex systems using cationic surfactants. While they are not useful for transfection, valuable information has been gathered about the phase behavior of DNA in these complexes. ${ }^{6-8}$ In complexes prepared from cationic lipids, initial structural investigations were carried out using transmission electron microscopy (TEM). ${ }^{9}$ Structures formed by cationic lipid-DNA complexes in the presence of a helper lipid, usually DOPE or DOPC, have been examined using SAXS ${ }^{10,11}$ and reviewed recently. ${ }^{12}$ These studies have been very useful in two respects. They have shown conclusively that X-ray diffraction is a good tool for the characterization of the structures of these and other complexes. They have also shown that these complexes condense DNA into ordered phases, which may be biologically significant since many viruses are known to have these higher levels of organization within their structures. Lamellar and inverted hexagonal lipid phases have been identified in these studies. ${ }^{10,13}$ It is the presence of the helper lipid, in increasing quantities, that initiates the lamellar-tohexagonal transition. It has been suggested, and some evidence exists, to say that inverted hexagonal lipid phases may be more successful in transfection, since they are more fusogenic and interact more strongly with cell membranes. The true significance of these investigations, however, may not be fully appreciated until we have a better understanding of the mechanisms by which efficient gene transfer is achieved.

Unlike cationic lipoplexes, there is no direct electrostatic interaction between DNA and zwitterionic lipids; however, the

* Corresponding author. Telephone: +3531 716 2300. Fax:+3531 716 2127. E-mail: kenneth@fiachra.ucd.ie.

${ }^{\dagger}$ University College Dublin.

₹ Ludwig Maximillian University. interaction can be mediated by divalent cations. Though it is known that polynucleotides can complex with zwitterionic lipids ${ }^{14}$ under these conditions, it has long been a common view that DPPC and other phosphatidylcholines bind only weakly to divalent cations. ${ }^{15}$ This may in part explain the reluctance to investigate these complexes in detail. However, there are advantages of using natural lipids in that they are completely non-cytotoxic. Although there is not a significant body of work completed on these complexes, DSC and temperature-scanning ultrasonic techniques have provided detailed information on the phase behavior of the zwitterionic lipid, both complexed and uncomplexed, and on the stoichiometry of the lipid in the complex. ${ }^{1,2,16}$

Here we study the complex formed from the interaction of DPPC, DNA, and calcium. The binding of calcium to zwitterionic lipids has been studied by a number of techniques, ${ }^{17}$ although the results have not been entirely consistent. Neutral lipids in the presence of divalent cations are capable of encapusulating linear and plasmid DNA, by simple selfassembly. The complex made with DOPC has been studied in some detail in this respect. ${ }^{18}$ Complexes formed with DNA, calcium, and egg lecithin exhibit a variety of structures. ${ }^{1}$ Lamellar and hexagonal lipid phases have been identified using freeze-fracture electron microscopy in these studies. Preliminary experiments using SAXS and electron microscopy have shown that well-ordered complexes made with DPPC, calf-thymus DNA, calcium, ${ }^{19}$ and magnesium ${ }^{20}$ and DOPC in the presence of DNA and manganese ${ }^{21}$ have a lamellar structure. A recent paper has reported the formation of a complex from only DNA and zwitterionic multilamellar vesicles with a small amount of added neutral cosurfactant. ${ }^{22}$ It has also been observed that DNA confined on a surface is condensed in the presence of divalent cations. $^{23}$

The study of DNA-calcium-lipid complexes is thus one of the interesting long-term strands of study in this field. Surprisingly, we report here that there appears to be a novel cooperative mechanism of DNA - calcium-zwitterionic lipid binding and earlier reservations about the weakness of the interaction may not limit the usefulness of the approach. We study the structure of complexes under varying conditions using small-angle X-ray scattering with a view to elucidating the binding mechanism. 


\section{Materials and Methods}

Materials and Sample Preparation. Calf-thymus DNA (Sigma) was purified by repeated phenol/chloroform extraction and precipitated in cold ethanol. The resulting pellet was dissolved in $10 \mathrm{mM}$ citrate buffer (pH 7.5) over a number of days and then dialyzed against $0.5 \mathrm{mM}$ HEPES for 3 days, with a change of buffer each day. This gave DNA with a 260/280 $\mathrm{nm}$ ratio greater than 1.8 , by UV absorbance.

DPPC (99\%) was purchased from Avanti Polar Lipids, (Alabaster, AL) and used without further purification. The dried lipid was dissolved in chloroform, and the solvent was removed under a stream of nitrogen. The dried film was then put in a vacuum oven overnight to remove any remaining solvent. Millipore water was added to give a lipid concentration of 25 $\mathrm{mg} / \mathrm{mL}$, and the lipid film was rehydrated at $50{ }^{\circ} \mathrm{C}$ for $2 \mathrm{~h}$. The lipid suspension was left at $4{ }^{\circ} \mathrm{C}$ under argon for 2 days before the samples were prepared. Multilamellar vesicles (MLVs) were prepared by vortexing the lipid suspension at $50{ }^{\circ} \mathrm{C}$. Small unilamellar vesicles (SUVs) were prepared by bath sonication at $50{ }^{\circ} \mathrm{C}$ to clarity.

Complexes for SAXS were prepared by mixing either the SUV or MLV lipid suspension and DNA in the wide part of a $1.5 \mathrm{~mm}$ X-ray capillary (Hilgenberg, Germany) to give an appropriate ratio. Calcium in HEPES buffer ( $\mathrm{pH} 7.5)$ was added, and the solution was mixed. The complex was left for $1 \mathrm{~h}$ at room temperature before being centrifuged into the narrow part of the capillary. The total volume in each capillary was always the same. The complexes were always prepared in an excess of water. Equilibration of the samples was carried out by varying the temperature between $25{ }^{\circ} \mathrm{C}$ and $55^{\circ} \mathrm{C}$ in a water bath, over at least 3 days. Samples were stored for at least 1 week before SAXS measurements were taken.

All buffer salts were analytical grade and were used without further purification.

Small-Angle X-ray Scattering. SAXS was carried out at both ID2 at the ESRF in Grenoble, France, and at A2 at the HASYLAB in Hamburg, Germany. The beam was calibrated with a silver behenate standard in each case. At ID2, the detector distance was $1.5 \mathrm{~m}$ and the wavelength of X-rays was $\sim 1 \AA$. Images were taken using a 2D detector. Samples run at ID2 were exposed for $100 \mathrm{~ms}$ at temperatures ranging from $25^{\circ} \mathrm{C}$ to $55^{\circ} \mathrm{C}$ in $1.5^{\circ} \mathrm{C}$ steps. The temperature was controlled by an external circulating water bath to $\pm 0.5^{\circ} \mathrm{C}$. The detector distance at A2 was $1.62 \mathrm{~m}$, and the wavelength was $1.5 \AA$. Exposure time at A2 was $120 \mathrm{~s}$ for each sample. No evidence of sample degradation was observed in any samples at this exposure. The results were recorded using a 1D linear detector.

\section{Results and Discussion}

Complexes were prepared from both multilamellar and unilamellar vesicles. Two distinct DPPC lamellar phases are present in both cases. ${ }^{1,2,16}$ These phases correspond to a DPPC phase bound to DNA and a second DPPC phase to which DNA is not bound. Both phases have been identified from the DSC analysis referenced above. Although samples were prepared at many different compositions, the results presented here are for samples prepared at a DNA/lipid mole ratio of 1:8, i.e., in excess lipid. Some of the samples were prepared at a number of different lipid concentrations (1:4 and 1:12). The results from those samples support the data presented in this work. However, the calcium concentration dependence was studied most systematically at the DNA/lipid mole ratio of 1:8, and therefore these data are used for the presentation here. The DNA concentration is expressed in moles per nucleotide throughout

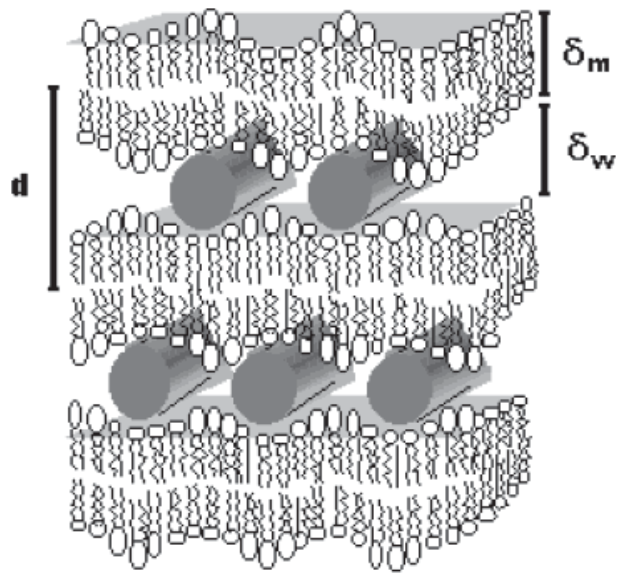

Figure 1. Expected structural arrangement of DPPC and DNA within the complex. The membrane width is indicated by $\delta_{\mathrm{m}}$, and the water spacing between the layer in which the DNA is accommodated is indicated by $\delta_{\mathrm{w}}$.

the present work. Due to differences in the mechanism of formation for complexes prepared from either ULVs or MLVs, both lamellar phases are present in the precipitate of the complex prepared from MLVs. Only the complexed phase of lipid is present in the precipitate in the unilamellar case, with the uncomplexed lipid in the supernatant. The mechanisms by which structural rearrangement of liposomes and DNA occurs to form these organized supramolecular structures are not yet fully understood. It is probable that fusion takes place on the DNA surface after mixing, eventually leading to the structures observed. Hayes et al. showed that, for this complex, fusion of liposomes on the DNA surface does take place and that it is liposome size dependent. ${ }^{24}$ The average size of the unilamellar liposomes prepared is about $100 \mathrm{~nm}$, which will probably result in complex formation occurring reasonably fast. In this case, the precipitated portion of the mixture separates from the excess material and, once centrifuged, lies in the lower portion of the $\mathrm{X}$-ray capillary with uncomplexed liposomes in the supernatant. Since only the precipitated portion of the lipid is placed in the $\mathrm{X}$-ray beam, only one lamellar phase is present in the SAXS pattern. Multilamellar vesicles by their nature are much larger, so the rate of fusion on the DNA surface is expected to be slower. We envisage that the DNA will initially bind to the outer layers of the MLVs, essentially "sticking" them together, which results in unbound lipid residing in the inner layers of the multilayered vesicles being precipitated with the DNA-bound lipid. All of this aggregate containing both lipid phases is then centrifuged to the end of the capillary during sample preparation. Intercalation of DNA to some of the internal layers of lipid will probably occur during the annealing process, which follows, but since the lipid is in excess, domains of uncomplexed lipid will still remain even after equilibration.

The DNA-bound lipid phase has a lamellar structure, a lattice of DNA layers, embedded in a DPPC lamellar phase, similar to that seen in cationic systems ${ }^{10}$ (Figure 1). Figure 2 and caption show a typical result for complexes prepared from MLVs. In this figure we show the series of Bragg reflections observed for both the DNA-bound lipid fraction and the uncomplexed lipid fraction. Furthermore, we show the data obtained for both lipid lamellar phases (bound and uncomplexed) when the lipid molecules in the bilayers are in (a) the gel and (b) the liquid crystalline states.

The lamellar repeat distance ( $d$ spacing) was calculated as $d$ $=2 \pi n / q$, where $n$ is the order of the Bragg peak. The value of $q$ for each sample was determined by fitting a Lorentz function 


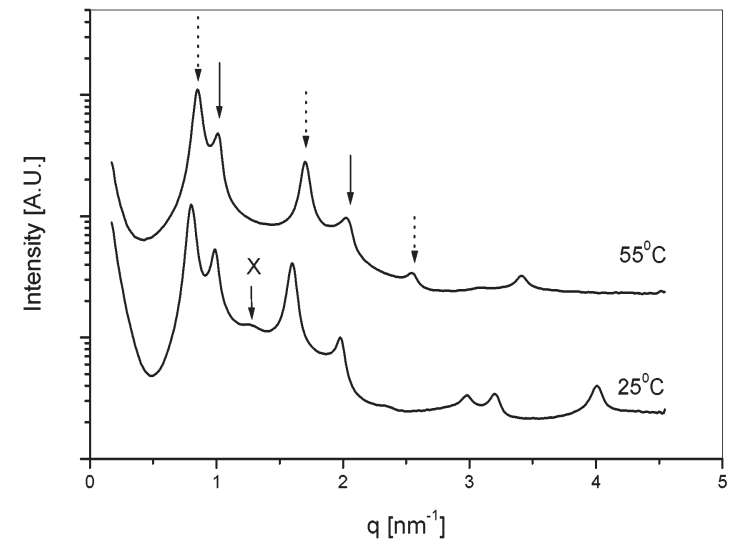

Figure 2. Intensity vs $q\left[\mathrm{~nm}^{-1}\right]$ for a complex prepared at a DNA/ lipid ratio of 1:8 from multilamellar vesicles in $5 \mathrm{mM} \mathrm{CaCl}_{2}$. The SAXS results for the two lipid lamellar phases described in the text in both the gel $\left(25^{\circ} \mathrm{C}\right)$ and liquid crystalline $\left(55^{\circ} \mathrm{C}\right)$ "thermotropic" phases are shown. The SAXS patterns of both lamellar phases exhibit separate sets of peaks for bound (DNA) and unbound lipid fractions. The dashed arrows indicate the Bragg peaks that correspond to the bound lipid phase, with $d=7.84 \mathrm{~nm}$. The full arrows indicate the unbound DPPC phase. A diffuse peak for DNA is seen at $25^{\circ} \mathrm{C}$, indicated by X. This corresponds to a DNA-DNA in-plane distance of $5.14 \mathrm{~nm}$, calculated as $d_{\mathrm{DNA}}=2 \pi / q_{\mathrm{DNA}}$.

to each peak. DPPC in HEPES was used as a control sample. The calculated lamellar repeat distance for this DPPC sample prepared in the absence of DNA and calcium in 5mM HEPES was $6.34 \mathrm{~nm}$ and is consistent with literature values for pure DPPC. ${ }^{25,26}$ Above the transition temperature $\left(41.3^{\circ} \mathrm{C}\right)$ of the uncomplexed lipid, this spacing increases to $6.72 \mathrm{~nm}$, again consistent with literature values. ${ }^{26}$ The lamellar repeat distance $d$ is the sum of the bilayer thickness $\delta_{\mathrm{m}}$ and the water spacing $\delta_{\mathrm{w}}$ between the bilayers, i.e., $d=\delta_{\mathrm{m}}+\delta_{\mathrm{w}}$ (Figure 1). This uncomplexed lipid exhibits the usual DPPC temperaturedependent phase behavior, here called "thermotropic phase behavior", with the $\mathrm{L}_{\beta^{\prime}}-\mathrm{P}_{\beta^{\prime}}-\mathrm{L}_{\alpha}$ phase transitions occurring as has been previously observed with the onset of the $\mathrm{P}_{\beta^{\prime}}$ or ripple phase at $\sim 34{ }^{\circ} \mathrm{C} .{ }^{27}$

It is our intention to describe the data obtained from both the DNA-bound lipid and the uncomplexed lipid phases separately at this point. It must be noted, however, that both of these lipid fractions, coexist within the same precipitate. The effect of calcium on the uncomplexed lipid is described first. At $25{ }^{\circ} \mathrm{C}$ with increasing calcium concentration, the lamellar repeat distance of the uncomplexed lipid within the precipitate increases until $\sim 5 \mathrm{mM} \mathrm{CaCl}_{2}$ is reached, after which it decreases slightly over the calcium concentration range studied (Figure 3). Above the transition temperature, however, a far larger effect is observed. Over the same calcium range, the DPPC repeat distance decreases from $6.72 \mathrm{~nm}$ in the absence of $\mathrm{CaCl}_{2}$ to $5.65 \mathrm{~nm}$ in $100 \mathrm{mM} \mathrm{CaCl}_{2}$ at $55^{\circ} \mathrm{C}$. This is contrary to what is seen in the DNA-free system. ${ }^{28,29}$ Above $1 \mathrm{mM} \mathrm{CaCl}_{2}$, the bilayers usually swell into excess water, until at much higher concentrations a lamellar structure with a repeat distance similar to that of pure DPPC re-appears due to screening of the bound calcium. We have repeated this work and observed the same effect (Figure 4). We believe that the unusual behavior we see is due to the uncomplexed lipid fraction being present within the same aggregate as the DNA-bound lipid fraction. We envisage that domains of uncomplexed lipid are present between domains of bound lipid, effectively "trapping" these domains within those of the bound lipid. Consequently the uncomplexed lipid is affected by the movements of the bound lipid phase. Even so, this does not entirely explain why the effect observed,

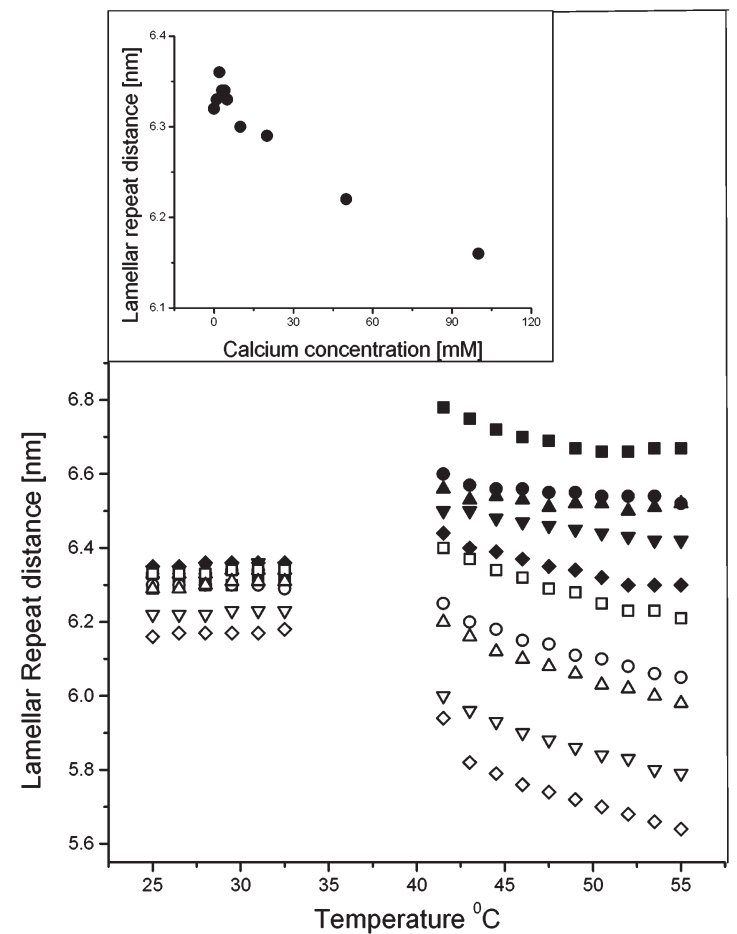

Figure 3. Lamellar repeat distance vs temperature for the coexisting uncomplexed lipid fraction (see Figure 2 and caption), resulting from a complex formed from multilamellar vesicles at varying concentrations

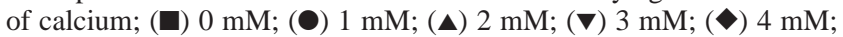

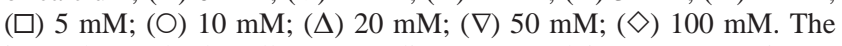
inset shows the lamellar repeat distance vs calcium concentration at $25^{\circ} \mathrm{C}$, indicating that initially there is a small increase in the lamellar repeat distance to $\sim 5 \mathrm{mM}$, after which the lamellar repeat distance decreases.

in the absence of DNA, on the lamellar repeat distance of the uncomplexed lipid in the presence of calcium is not observed here. Another factor is also at play. The method by which the samples are prepared is very important. In the work of Lis et al., ${ }^{28}$ calcium solutions $(30 \% \mathrm{w} / \mathrm{w})$ are added to dried lipid powder. The multilamellar structures are then formed in the presence of calcium. Our preparation method involves preparing multilamellar vesicles in water first, then adding DNA and then calcium. In order for calcium to be present in internal layers of lipid, ions must pass through the hydrophobic interior of the lipid membranes. This ion transport is entropically unfavorable. Some calcium will probably be transferred to the internal layers during the annealing process, since the bilayer structures are disturbed, but it is more likely that calcium will bind to MLV surface layers and DNA first.

In the presence of DNA and calcium, the DPPC lamellar spacing increases to $7.84 \mathrm{~nm}$ at $25{ }^{\circ} \mathrm{C}$ in $20 \mathrm{mM} \mathrm{CaCl}_{2}$ for complexes prepared from MLVs. This is consistent with the value determined for the corresponding complex prepared from ULVs $(7.89 \mathrm{~nm})$. This is an increase of $1.5 \mathrm{~nm}$ on the lamellar repeat distance for DPPC alone $(6.34 \mathrm{~nm})$. If the bilayer thickness is taken as $4.34 \mathrm{~nm}$ (value for DPPC at $20{ }^{\circ} \mathrm{C}{ }^{25}$ ), there is adequate room for the diameter of a hydrated $\mathrm{B}-\mathrm{DNA}$ strand. ${ }^{30}$ This bound DPPC has a transition temperature higher than free lipid at $\sim 43{ }^{\circ} \mathrm{C}$, which has also been observed by both DSC and temperature-scanning ultrasound. 1,2,16

In the MLV case, increasing the concentration of calcium from $1 \mathrm{mM}$ to $4 \mathrm{mM}$ increases the bound DPPC lamellar repeat distance slightly from $7.85 \mathrm{~nm}$ in $1 \mathrm{mM} \mathrm{CaCl}_{2}$ to $7.88 \mathrm{~nm}$ in 4 $\mathrm{mM} \mathrm{CaCl} 2$ at $25^{\circ} \mathrm{C}$, which after $5 \mathrm{mM} \mathrm{CaCl}_{2}$ begins to increase again (Figure 5). At $5 \mathrm{mM} \mathrm{CaCl}_{2}$ there is a minimum in the 

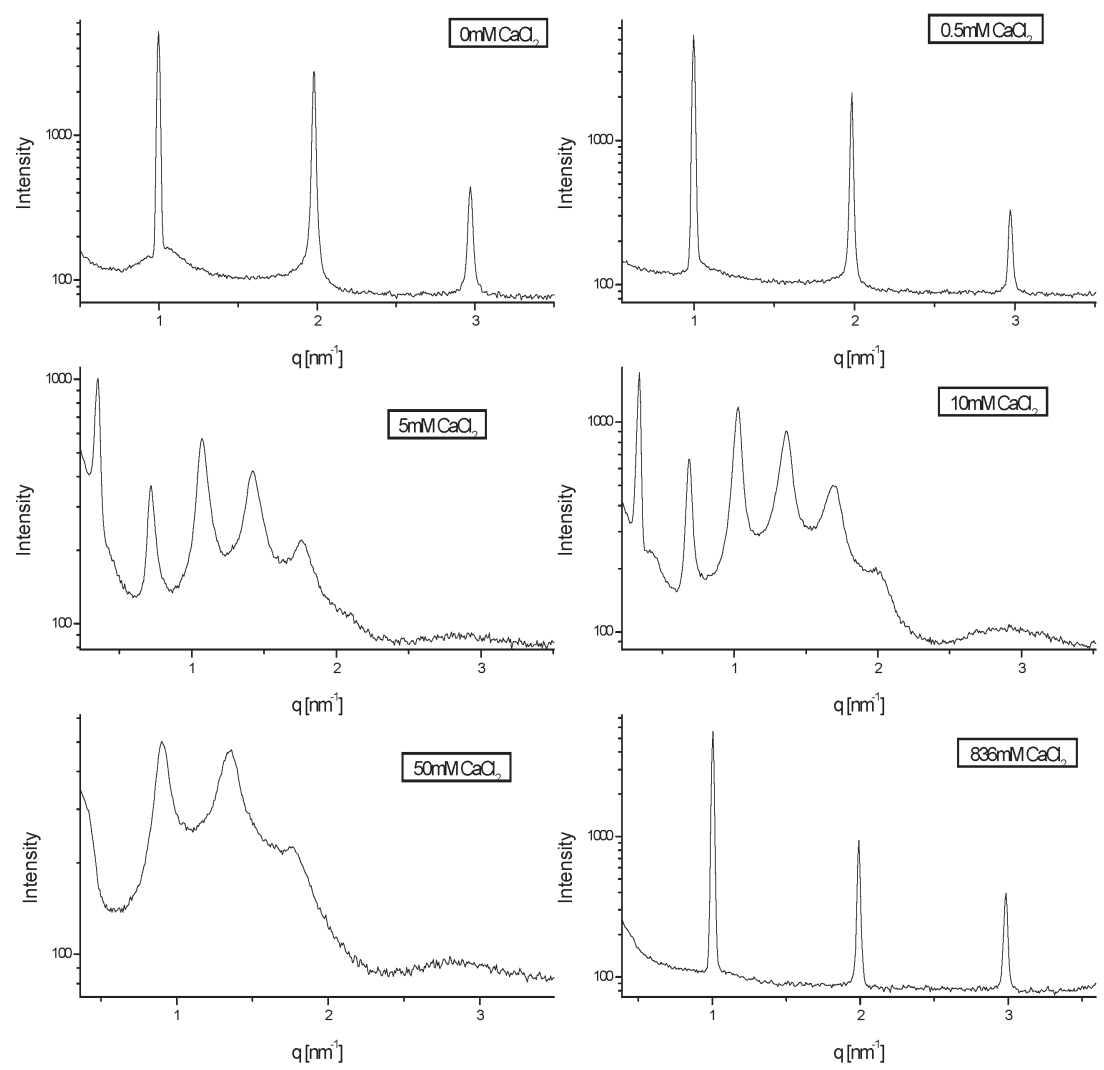

Figure 4. Effect of calcium on DPPC multilayers at varying calcium concentrations in the absence of DNA. This is a repetition of some of the work of Lis et al. ${ }^{29}$ Samples are prepared by mixing $30 \mathrm{wt} \%$ DPPC with either water or calcium solution, as indicated, and equilibrating over a number of days before SAXS measurements are taken. At low calcium concentration, the lamellar structure usually observed for DPPC in water exists. Beyond this point the DPPC layers swell into excess water, until at much higher concentrations (836 mM shown here), a lamellar structure similar to the one observed in the low calcium case is observed.

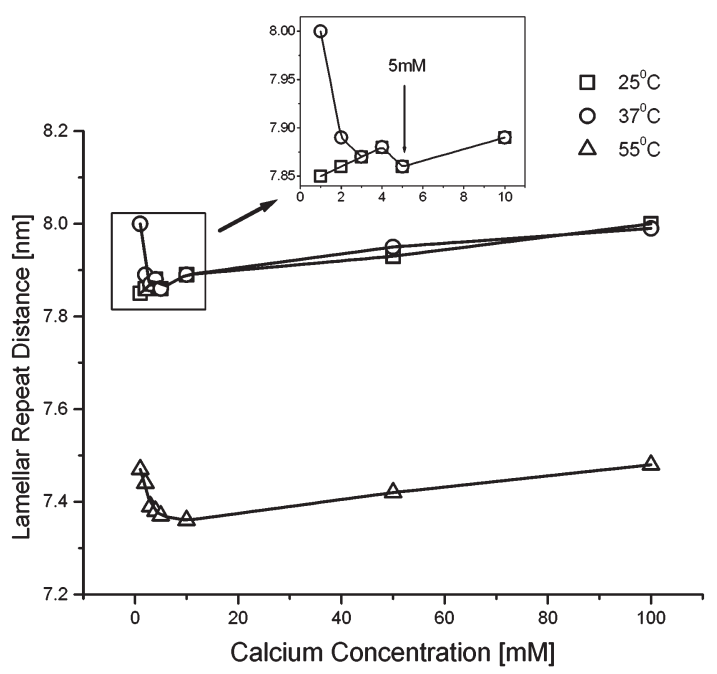

Figure 5. Lamellar repeat distance vs calcium concentration for bound lipid in complexes prepared from multilamellar vesicles (same conditions as in Figure 2).

lamellar repeat distance of $7.86 \mathrm{~nm}$. This is different from the effect observed at $55^{\circ} \mathrm{C}$, although a minimum at $5 \mathrm{mM}$ is also observed. The lamellar repeat distance calculated at $1 \mathrm{mM} \mathrm{CaCl}_{2}$ in the $\mathrm{L}_{\alpha}^{C}$ phase is $7.47 \mathrm{~nm}$, after which there is a decrease in lamellar repeat distance with a minimum between 5 and $10 \mathrm{mM}$ $\mathrm{CaCl}_{2}$ at $7.37 / 7.36 \mathrm{~nm}$. These complexes appear to be well ordered with 5 Bragg peaks being observed in most samples. Above the transition temperature of this phase, four Bragg peaks are still observed. What is very clear from these results and from the corresponding ones for the complexes prepared from

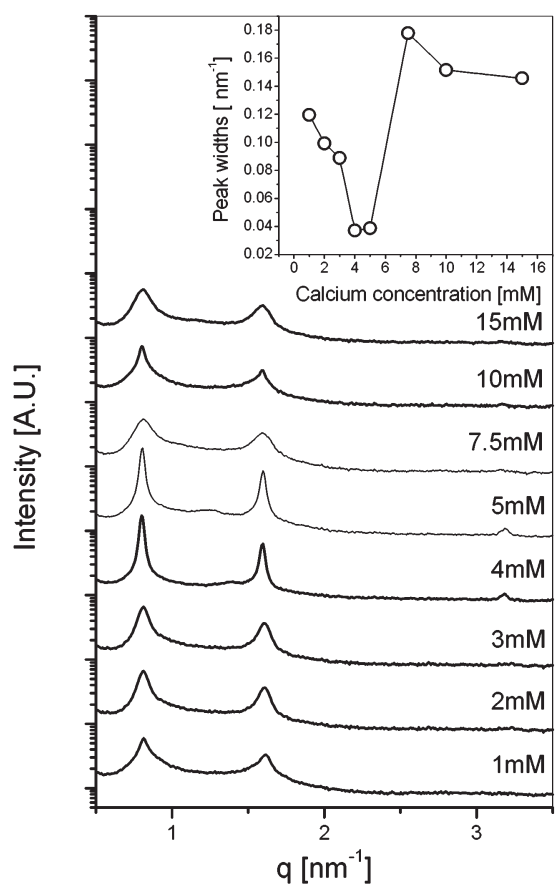

Figure 6. Intensity vs $q\left[\mathrm{~nm}^{-1}\right]$ for complexes prepared at a DNA/ lipid mole ratio of 1:8, with increasing calcium concentration. The most notable difference is the change in peak shape. Significant narrowing of the Bragg peaks is observed at $4-5 \mathrm{mM} \mathrm{CaCl}_{2}$ (inset).

ULVs is the significant narrowing of the Bragg peaks between 4 and $5 \mathrm{mM} \mathrm{CaCl}_{2}$ (Figure 6). This narrowing has been quantified by fitting Lorentz functions to each of the peaks. The SAXS for complexes prepared from ULVs has been shown 
for comparison, but this narrowing is observed in both sets of data.

From these observations, it is our intention to determine the way in which calcium binds to DPPC in our system. Calcium binds weakly to DPPC membranes, ${ }^{15}$ and it is clear from many studies that binding of $\mathrm{Ca}^{2+}$ to DPPC occurs via the phosphate moiety on the headgroup portion of the lipid. The binding of calcium to DPPC within our complex is expected to be much stronger, since increased binding of calcium to PC membranes in complexes with negatively charged polyelectrolytes has been observed. ${ }^{31}$ These researchers measured binding constants for calcium in a complex formed from dextran sulfate and DMPC. They verified that the binding of calcium in the lipid-DS complex was significantly enhanced. The headgroup portion of DMPC is identical to our lipid, and dextran sulfate being a negatively charged polyelectrolyte, albeit a more flexible one than DNA, should bind in a similar way.

Evidence of zwitterionic lipid participation in DNA binding has already been suggested. Researchers have looked at mixtures of DMTAP and DMPC. ${ }^{32,33}$ These lipids are chemically similar apart from the phosphate moiety present in the zwitterionic form. Pressure-area isotherms constructed for each lipid and various compositions of the two revealed a minimum in headgroup area at an equimolar ratio of the two lipids, suggesting involvement of the zwitterionic headgroup in DNA binding.

In summary, it appears that $5 \mathrm{mM} \mathrm{CaCl}_{2}$ is a special point at which some compact structural arrangement is reached. We will refer to this point as $C^{*}$. This is the minimum in lamellar repeat distance for the DNA-bound lipid within the complex formed from MLVs. The uncomplexed lipid domains trapped within this complex structure aggregate are also affected at this concentration. There is a sharp increase in the lamellar repeat distance observed above the transition temperature for the uncomplexed lipid (Figure 3). This is a direct observation of the uncomplexed lipid phase being directly affected by the bound lipid. At concentration $C^{*}$, there is a minimum in Bragg peak width for the bound lipid (Figure 6). This is probably the most important observation. A decrease in the peak width is indicative of increased order of the lipid molecules within the bilayers. This minimum is observed for complexes prepared from MLVs and ULVs. This is also the calcium concentration at which a DNA-DNA in-plane correlation is observed (Figure 2).

What is special about $C^{*}$ ? We now consider the stoichiometry of the complex. The concentration of lipid in all of the samples is $22.7 \mathrm{mM}$. Since the complexes are prepared at a DNA/lipid mole ratio of 1:8, the concentration of DNA is then $2.8 \mathrm{mM}$ (nucleotides). The estimated number of strongly bound lipid molecules per DNA phosphate in this complex is $4.5-5 .^{2}$ Assuming there are 5 strongly bound DPPC molecules for each DNA phosphate and that calcium binds preferentially to DPPC within a complex, concentration $C^{*}$ corresponds to a point in these samples where there is roughly $1 \mathrm{CaCl}_{2}$ for every two DPPC molecules. This suggests that calcium bridges two neighboring lipid molecules through their phosphate groups, thereby neutralizing the negative charge on each lipid. This leads to a cationic moiety in the binding to DNA. This would be achieved by a reorientation of the headgroup of the lipid, effectively leaving a positive charge on every DPPC.

There are two possibilities for $\mathrm{PC}-\mathrm{Ca}^{2+}-$ DNA binding in this complex. Both options are indicated schematically in Figure 7. In water, the headgroups of DPPC are mainly orientated, with the phosphate closest to the lipid/water interface and the trimethylamine portion of the lipid orientated downward to the

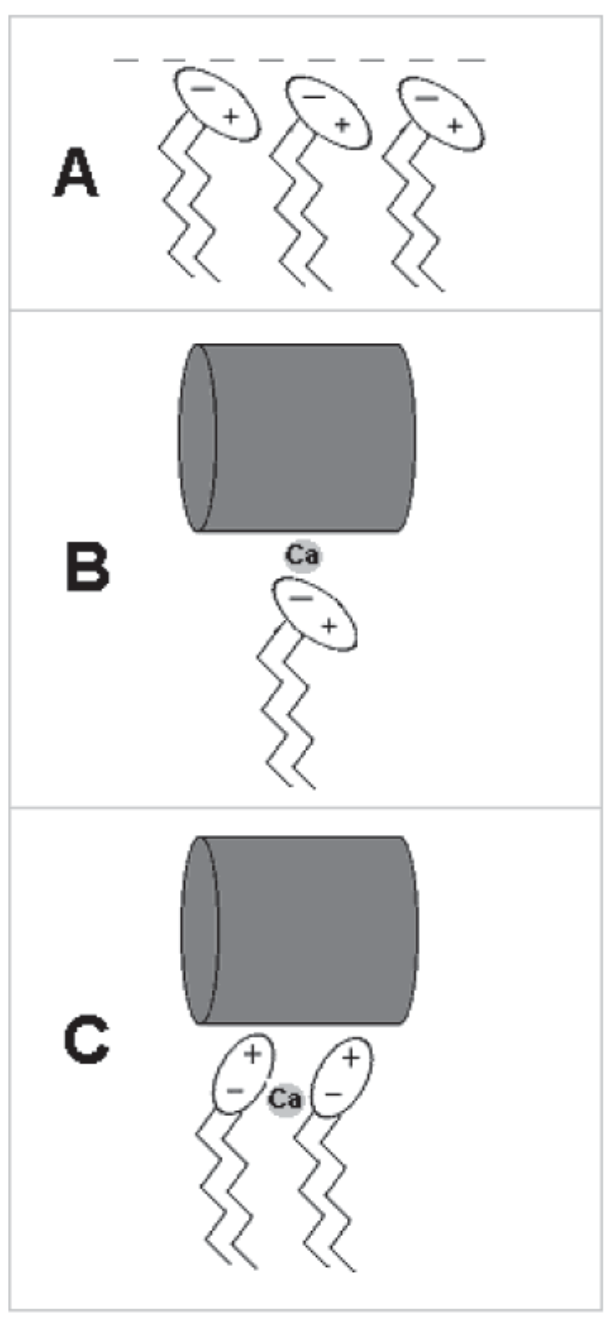

Figure 7. (A) Schematic picture of the orientation of DPPC lipids in water. (B) Option 1 in the binding of DPPC to DNA via calcium. The calcium ion bridges the phosphate of the lipid to the phosphate of the DNA. (C) Option 2: The calcium ion bridges two adjacent DPPC molecules, leaving a net positive charge on each which then binds to DNA. (Diagrams are not drawn to scale and are for illustration purposes only.)

hydrocarbon tails (Figure 7a). The first option in DNA binding in the presence of calcium would be for calcium to bridge the phosphate on the lipid headgroup with a phosphate on DNA (Figure 7b). The second option, which is the one we believe to be true, is a reorientation in the presence of calcium leaving the headgroups of the DPPC with the positively charged amine pointing toward the DNA (Figure 7c). This is identical to the portion of lipid involved in DNA binding in any closely related TAP cationic lipid-DPTAP, 1,2-dipalmitoyl-3-trimethylammonium-propane is the most closely related cationic lipid to DPPC. The most convincing evidence for this argument is the narrowing of the Bragg peak at concentration $C^{*}$ in complexes prepared from both ULVs and MLVs. If a reorientation of the headgroup does occur, the narrowing of the Bragg peak indicates a minimum in membrane fluctuations at concentration $C^{*}$, therefore appearing as increased order in the SAXS pattern (Figure 6).

Other evidence for this supposition exists. Inclusion of cholesterol in the bilayer decreases the affinity of $\mathrm{Ca}^{2+}$ for PC membranes. ${ }^{34}$ This is also the case for our complex. With increasing cholesterol content, the partitioning of DNA into an aqueous phase from an inverse micellar phase in organic solvent increases. ${ }^{35}$ Effectively, complex formation is inhibited by 
cholesterol. A reasonable explanation for this would be that calcium binding to DPPC occurs only when the molecules are in close proximity to each other. Cholesterol inserts between the tails of the phospholipid, separating the headgroups. We believe, then, that DPPC molecules are more likely to bind to each other via calcium and then to DNA in our complex than to bind a single $\mathrm{Ca}^{2+}$ to each DPPC and bridge the lipid to the DNA phosphate, as suggested by Budker et al. ${ }^{14}$

The structural arrangement that we propose is based on results obtained from equilibrated samples. The mechanisms by which these and cationic lipoplexes form are still to be confirmed, although some work has been done. ${ }^{36}$ Binding of the lipid to DNA must first occur, followed by lipid fusion on the DNA surface and then some structural reorganization. We know that in this complex lipid fusion is induced in the presence of DNA. ${ }^{24}$ The structural arrangement of calcium in any intermediate step may differ from the arrangement we propose. Initially, calcium is far more likely to bind to DNA. It is easy to envisage that the arrangement shown in Figure 7b, might well occur in the early stages of complex formation. Structural rearrangement would facilitate the picture we propose. Further work will be required to elucidate the mechanism of formation further.

\section{Conclusions}

The main conclusions of this study are as follows. DNA and DPPC in the presence of calcium form a complex with a welldefined multilamellar structure. Zwitterionic PC lipids, DPPC in particular, long considered to have only weak interactions to DNA in the presence of calcium, under the appropriate conditions can in fact interact strongly. This enhancement is associated with an arrangement of calcium, DNA, and lipid in which the calcium and lipid form a new bridging unit and acquire effective "cationic" properties, without the consequent toxicity. This we know from observing a minimum in the observed lamellar repeat distance at a concentration $C^{*}$. Also at this concentration there is a decrease in the Bragg peak width, indicating increased membrane order. $C^{*}$ corresponds to a point where there is one calcium atom for 2 DPPCs if we assume there are 4.5-5 bound lipid molecules per phosphate, which was calculated by Kharakoz et al. ${ }^{2}$ The resulting complexes lead to a similar range of lamellar "sandwich" type structures that have been observed in cationic-DNA systems, again supporting the view that in situ the lipid-calcium system is acting as an effective cationic. The resources devoted to creating cationicDNA complexes that are less toxic, may perhaps, in future be balanced by research exploiting the possibility of creating comparable complexes from entirely nontoxic components such as those outlined above.

Acknowledgment. The authors acknowledge experimental support from staff at the synchrotron facilities, Dr. T. Narayanan on ID2 at the ESRF and Dr. Sergio Funari on A2 at HASYLAB. We also acknowledge experimental input from Dr. Franck Artzner, and thank him for providing the apparatus for the temperature control experiments. The authors acknowledge the Max Planck Institute for Polymer Research, Mainz, Germany. It was during time spent there that some of the results included in this paper were obtained. J. J. McManus acknowledges financial support from Enterprise Ireland.

\section{References and Notes}

(1) Tarahovsky, Y. S.; Khusainova, R. S.; Gorelov, A. V.; Nicolaeva, T. I.; Deev, A. A.; Dawson, K. A.; Ivanitsky, G. R. DNA initiates polymorphic structural transitions in lecithin. FEBS Lett. 1996, 390, 133.
(2) Kharakoz, D. P.; Khusainova, R. S.; Gorelov, A. V.; Dawson, K. A. Stoichiometry of dipalmitoylphosphatidylcholine-DNA interaction in the presence of $\mathrm{Ca}^{2+}$ : a temperature-scanning ultrasonic study. FEBS Lett. 1999, 446, 27.

(3) Chesnoy, S.; Huang, L. Structure and Function of Lipid-DNA Complexes for Gene Delivery. Annu. Rev. Biophys. Biomol. Struct. 2000, 29, 27.

(4) Felgner, P. L.; Gadek, T. R.; Holm, M.; Roman, R.; Chan, H. W.; Wenz, M.; Northrop, J. P.; Ringold, G. M.; Danielsen, M. Lipofection: A highly efficient, lipid-mediated DNA-transfection procedure. Proc. Natl. Acad. Sci. U.S.A. 1987, 84, 7413.

(5) Felgner, J. H.; Kumar, R.; Sridhar, C. N.; Wheeler, C. J.; Tsai, Y. J.; Border, R.; Ramsey, P.; Martin, M.,; Felgner, P. L. Enhanced Gene Delivery and Mechanism Studies with a Novel Series of Cationic Lipid Formulations. J. Biol. Chem. 1994, 269 (4), 2550.

(6) Dias, R.; Mel'nikov, S.; Lindman, B.; Miguel, M. G. DNA phase behavior in the presence of oppositely charged surfactants. Langmuir 2000, 16, 9577.

(7) Mel'nikov, S. M.; Khan, M. O.; Lindman, B.; Jonsson, B. Phase behavior of single DNA in mixed solvents. J. Am. Chem. Soc. 1999, 121, 1130.

(8) Dias, R.; Antunes, F.; Miguel, M.; Lindman, S.; Lindman, B. DNAlipid systems. A physical chemistry study. Braz. J. Med. Biol. Res. 2002, 35, 509.

(9) Gustafsson, J.; Arvidson, G.; Karlsson, G.; Almgren, M. Complexes between cationic liposomes and DNA visualized by cryo-TEM. Biochim. Biophys. Acta 1995, 1235, 305.

(10) Rädler, J. O.; Koltover, I.; Salditt, T.; Safinya, C. R. Structure of DNA-Cationic Liposome Complexes: DNA Intercalation in Multilamellar Membranes in Distinct Interhelical Packing Regimes. Science 1997, 275, 810.

(11) Lasic, D. D.; Strey, H.; Stuart, M. C. A.; Podgornik, R.; Frederik, P. M. The Structure of DNA-Liposome Complexes. J. Am. Chem. Soc. 1997, 119, 832.

(12) Safinya, C. R. Structures of lipid-DNA complexes: supramolecular assembly and gene delivery. Curr. Opin. Struct. Biol. 2001, 11 (4), 440.

(13) Koltover, I.; Salditt, T.; Rädler, J. O.; Safinya, C. R. An Inverted Hexagonal Phase of Cationic Liposome-DNA Complexes Related to DNA Release and Delivery. Science 1998, 281, 78.

(14) Budker, V. G.; Godovikov, A. A.; Naumova, L. P.; Slepneva, I. A. Interaction of polynucleotides with natural and model membranes. Nucleic Acid Res. 1980, 8 (11), 2499.

(15) Marra, J.; Israelachvili, J. Direct Measurements of Forces between Phosphatidylcholine and Phosphatidylethanolamine Bilayers in Aqueous Electrolyte Solutions. Biochemistry 1985, 24, 4608.

(16) Tarahovsky, Y. S.; Deev, A. A.; Masulis, I. S.; Ivanitsky, G. R. Structural Organization and Phase Behavior of DNA-Calcium-Dipalmitoylphosphatidylcholine Complex. Biochem. (Moscow) 1998, 63 (10), 1126.

(17) Satoh, K. Determination of binding constants of $\mathrm{Ca}^{2+}, \mathrm{Na}^{+}$, and $\mathrm{Cl}^{-}$ions to liposomal membranes of dipalmitoylphosphatidylcholine at gel phase by particle electrophoresis. Biochim. Biophys. Acta 1995, 1239, 239, and references therein.

(18) Bailey, A. L.; Sullivan, S. M. Efficient encapsulation of DNA plasmids in small neutral liposomes induced by ethanol and calcium. Biochim. Biophys. Acta 2000, 1468, 239.

(19) Hayes, M. E.; Gorelov, A. V.; Dawson, K. A. Unpublished results.

(20) Uhríková, D.; Rapp, G.; Balgavý, P. Challenges for Coordination Chemistry in the New Century; Slovac Technical University Press: pp 219224.

(21) Francescangeli, O.; Stanic, B.; Gobbi, L.; Bruni, P.; Iacussi, M.; Tosi, G.; Bernstoff, S. Structure of self-assembled liposome-DNA-metal complexes. Phys. Rev. E. 2003, 67, 011904.

(22) Pott, T.; Roux, D. DNA intercalation in neutral multilamellar membranes. FEBS Lett. 2002, 511 (1-3), 150

(23) Koltover, I.; Wagner, K.; Safinya, C. R. DNA condensation in two dimensions. Proc. Natl. Acad. Sci. 2000, 97, 14046.

(24) Hayes, M. E.; Gorelov, A. V.; Dawson, K. A. DNA-induced fusion of phosphatidylcholine vesicles. Prog. Colloid Polym. Sci. 2001, 118, 243.

(25) Sun, W. J.; Tristam-Nagle, S.; Suter, R. M.; Nagle, J. F. Structure of Gel Phase Saturated Lecithin Bilayers: Temperature and Chain Length Dependence. Biophys. J. 1996, 71, 885.

(26) Nagle, J. F.; Zhang, R.; Tristram-Nagle, S.; Sun, W.; Petrache, H. I.; Suter, R. M. X-ray Structure Determination of Fully Hydrated $L_{\alpha}$ Phase Dipalmitoylphosphatidylcholine Bilayers. Biophys. J. 1996, 70, 1419.

(27) Nagle, J. F.; Tristam-Nagle, S. Structure of lipid bilayers. Biochim. Biophys. Acta 2000, 1469, 159.

(28) Lis, L. J.; Lis, W. T.; Parsigian, V. A.; Rand, R. P. Adsorption of Divalent Cations to a Variety of Phosphatidylcholine Bilayers. Biochemistry 1981, 20, 1771.

(29) Lis, L. J.; Parsegian, V. A.; Rand, R. P. Binding of Divalent Cations to Dipalmitoylphosphatidylcholine Bilayers and Its Effect on Bilayer Interaction. Biochemistry 1981, 20, 1761. 
(30) Podgornik, R.,; Rau, D. C.; Parsegian, V. A. The Action of Interhelical Forces on the Organization of DNA Double Helices: Fluctuation-Enhanced Decay of Electrostatic Double-Layer and Hydration Forces. Macromoecules 1989, 22, 1780.

(31) Huster, D.; Arnold, K. Ca ${ }^{2+}$ - Mediated Interaction between Dextran Sulfate and Dimyristoyl-sn-Glycero-3-Phosphocholine Surfaces Studied by ${ }^{2} \mathrm{H}$ Nuclear Magnetic Resonance. Biophys. J. 1998, 75, 909.

(32) Zantl, R.; Baicu, L; Artzner, F.; Sprenger, I.; Rapp, G.; Rädler, J. O. Thermotropic Phase Behavior of Cationic Lipid-DNA Complexes Compared to Binary Lipid Mixtures. J. Phys. Chem. B 1999, 103, 10300.
(33) Bandyopadhyay, S.; Tarek, M.; Klein, M. L. Molecular Dynamics Study of a Lipid-DNA Complex. J. Phys. Chem. B 1999, 103, 10075.

(34) Inoko, Y.; Yamaguchi, T.; Furuya, K.; Mitsui, T. Effects of Cations on Dipalmitoyl Phosphatidylcholine/Cholesterol/Water Systems. Biochim. Biophys. Acta 1975 413, 24.

(35) Gorelov, A. V.; Hayes, M. E.; Wehling, A.; Dawson,K. A. Il Nuovo Cimento D 1998, 20, 2401.

(36) Barreleiro, P. C. A.; May, R. P.; Lindman, B. Mechanism of Formation of DNA-Cationic Vesicle Complexes. Faraday Discuss. 2003, 122, 191. 